Nonlinear Processes in Geophysics, 12, 979-991, 2005

SRef-ID: $1607-7946 / \mathrm{npg} / 2005-12-979$

European Geosciences Union

(c) 2005 Author(s). This work is licensed

under a Creative Commons License.

\title{
Testing the performance of three nonlinear methods of time series analysis for prediction and downscaling of European daily temperatures
}

\author{
J. Miksovsky and A. Raidl \\ Dept. of Meteorology, Faculty of Mathematics and Physics, Charles University, Prague, Czech Republic \\ Received: 16 June 2005 - Revised: 15 September 2005 - Accepted: 15 September 2005 - Published: 9 November 2005
}

\begin{abstract}
We investigated the usability of the method of local linear models (LLM), multilayer perceptron neural network (MLP NN) and radial basis function neural network (RBF NN) for the construction of temporal and spatial transfer functions between different meteorological quantities, and compared the obtained results both mutually and to the results of multiple linear regression (MLR). The tested methods were applied for the short-term prediction of daily mean temperatures and for the downscaling of NCEP/NCAR reanalysis data, using series of daily mean, minimum and maximum temperatures from 25 European stations as predictands. None of the tested nonlinear methods was recognized to be distinctly superior to the others, but all nonlinear techniques proved to be better than linear regression in the majority of the cases. It is also discussed that the most frequently used nonlinear method, the MLP neural network, may not be the best choice for processing the climatic time series LLM method or RBF NNs can offer a comparable or slightly better performance and they do not suffer from some of the practical disadvantages of MLPs.

Aside from comparing the performance of different methods, we paid attention to geographical and seasonal variations of the results. The forecasting results showed that the nonlinear character of relations between climate variables is well apparent over most of Europe, in contrast to rather weak nonlinearity in the Mediterranean and North Africa. No clear large-scale geographical structure of nonlinearity was identified in the case of downscaling. Nonlinearity also seems to be noticeably stronger in winter than in summer in most locations, for both forecasting and downscaling.
\end{abstract}

Correspondence to: J. Miksovsky

(jiri.miksovsky@mff.cuni.cz)

\section{Introduction}

Within the last two decades, numerous new methods of time series analysis have been developed for dealing with nonlinear data (see, e.g. Abarbanel, 1996; Kantz and Schreiber, 1997; Haykin, 1999; Galka, 2000 for an overview), and a lot of them have found their place in the study of meteorological signals (see examples in Sect. 4). But it has also been shown that application of nonlinear methods does not automatically grant better results than use of their linear counterparts (e.g. Tang et al., 2000), despite the fact that the meteorological series originate from an inherently nonlinear system. Application of nonlinearity tests reveals some climatic data sets to appear linear (Palus and Novotna, 1994; Schreiber and Schmitz, 2000; Miksovsky and Raidl, 2005), while others may exhibit nonlinear characteristics (Palus and Novotna, 1994; Palus, 1996; Tsonis, 2001; Miksovsky and Raidl, 2005). In this paper, our intention was to address the problem of nonlinearity of the atmospheric time series from a rather practical point of view and to ascertain the performance of several nonlinear methods of time series analysis for two typical meteorological problems: construction of temporal (prediction) and spatial (downscaling) mappings at synoptic time scales. The examined methods included a nonlinear technique which has already become common in the atmospheric sciences (multilayer perceptron neural network - MLP NN), as well as methods which are less common, at least to date - local linear models in the reconstructed phase space (LLM) and radial basis function neural networks (RBF NN). Performance of nonlinear methods was compared both to each other and to the results of multiple linear regression (MLR). Aside from presenting examples of the obtained results, including their spatial and seasonal variances, we have tried to draw conclusions about the tested methods' disadvantages and strong points, as well as the pros and cons associated with their implementation. 


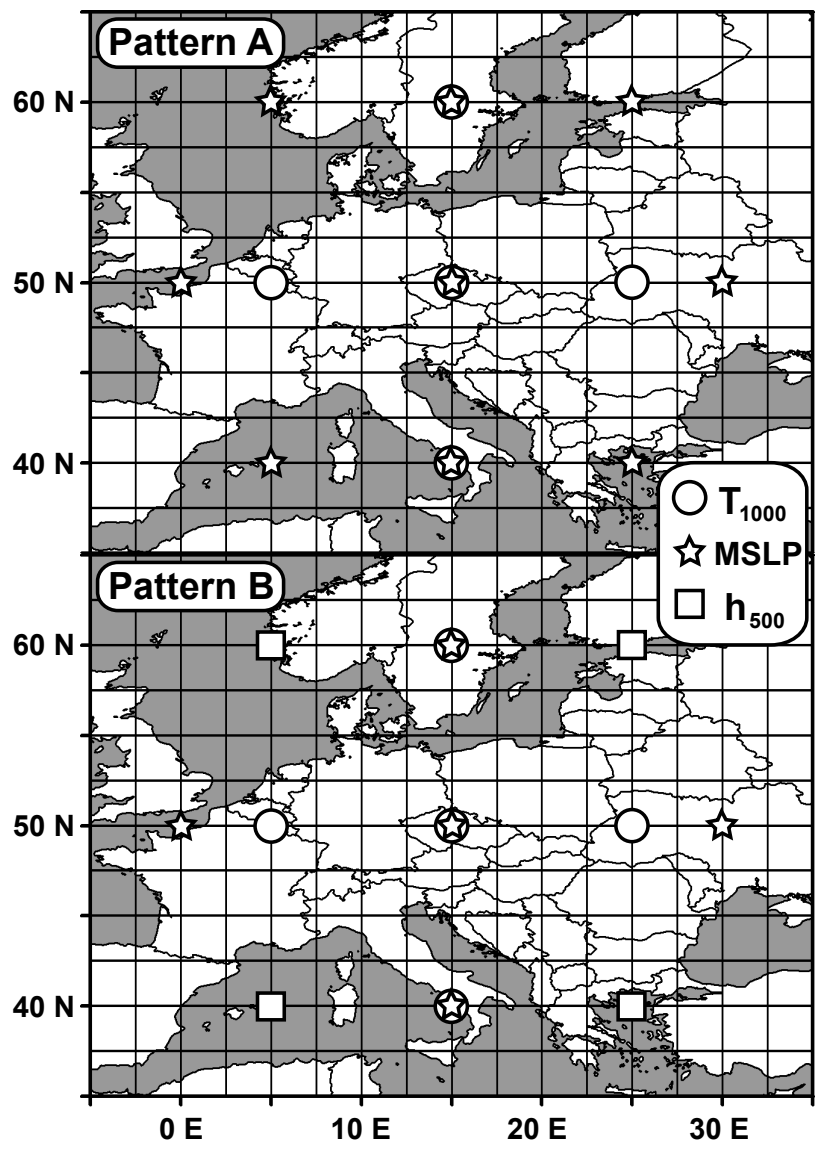

Fig. 1. The pattern of predictors used in the case of prediction (Pattern A) and downscaling (Pattern B), displayed for the predictand series located at $50^{\circ} \mathrm{N}, 15^{\circ} \mathrm{E}$. The grid of horizontal and vertical lines illustrates the full resolution of the NCEP/NCAR reanalysis data set.

\section{Methods}

\subsection{Choice of predictors}

The first problem which needs to be addressed before time series analysis methods can be applied is the issue of the structure of the predictor space. The climate system, as well as its local subsystems, has an infinite number of degrees of freedom, meaning that an infinite number of variables would be required for its state exact description. But there are methods by which the state can be characterized, at least approximately and locally, in a relatively low number of quantities. Techniques referred to as phase space (PS) reconstruction represent a way of achieving such a description. The most classical method of phase space reconstruction, time delay reconstruction from a scalar series (Packard et al., 1980; Takens, 1981), has been applied for analysis of climatic time series at a number of occasions, from early attempts to discover some kind of climate attractor (among many e.g. Fraedrich, 1986; Keppenne and Nicolis, 1989), to its practical implementations for the forecast of meteorological or hydrological variables (e.g. Abarbanel, 1996;
Pérez-Muñuzuri and Gelpi, 2000; Jayawardena and Gurung, 2000). Many more examples from various climate-related disciplines can be found in the paper by Sivakumar (2004). But it also turns out that the information content in a single time series is not always sufficient for the climate system's state characterization. This is especially true when the nonlinear component of the analyzed signal is to be studied (Miksovsky and Raidl, 2005). Fortunately, meteorological measurements (or numerical model outputs, reanalyses and similar data sets) are typically available for several variables and in numerous locations, which allows for the use of multivariate phase space reconstruction (Keppenne and Nicolis, 1989). The vector in the reconstructed phase space, characterizing the system's state in time $t$ (and representing the vector of predictors), is denoted $\boldsymbol{y}(t)$ here,

$\boldsymbol{y}(t)=\left(X_{1}(t), X_{2}(t), \ldots, X_{m}(t)\right), t=1, \ldots, L$,

where $m$ characterizes the dimension of the reconstructed PS and is usually referred to as the embedding dimension, $L$ is the length of the series and $X_{i}(t), i=1, \ldots, m$, are elements of $\boldsymbol{y}(t)$. For multivariate reconstruction from $m$ scalar series $x_{i}(t)$, elements of $\boldsymbol{y}(t)$ take the following simple form:

$X_{i}(t)=x_{i}(t), i=1, \ldots, m$.

An important issue is the choice of suitable predictor series, i.e. specifying which series, and how many, will be used in the role of $x_{i}(t)$. We used NCEP/NCAR reanalysis series as potential predictors here, meaning that thousands of series of different quantities from numerous grid points and pressure levels were available, whereas only a few predictors were needed. Choice of predictors is a nontrivial problem and it can be done in several ways such as using step-wise regression or reducing the dimensionality of the predictor space by means of principal component analysis, but no approach can generally be considered to be the absolutely best one. Also, use of different sets of predictors may sometimes result in quite different outcomes, as shown by Huth (2004) for temperature downscaling. We have tested several methods for predictors selection and decided to utilize a pre-set pattern of input variables, consisting of values of $T_{1000}$, MSLP and $h_{500}$ from different grid points (see Sect. 3 for data sets description). Use of the pre-set pattern is fast and easy to implement; it does not favor the MLR method like the use of the stepwise linear regression could and it also gave better results (in terms of root mean squared error - RMSE) than use of the principal components as predictors, similarly to the findings of Huth (2002). Moreover, using the same type and number of predictors for different tested methods, locations and seasons makes intercomparison of the results easier, because the composition of the predictor space need not be taken into account as one of the variables. The patterns used for prediction (Sect. 4.1) and downscaling (Sect. 4.2) both had dimension $m=14$; their structure is shown in Fig. 1 . 


\subsection{Methods used}

Mapping from the predictor vector $\boldsymbol{y}(t)$ to predictand $x^{P R E D}(t)$ (transfer function) can be generally expressed as

$x^{P R E D}(t)=\Phi(\boldsymbol{y}(t))=\Phi\left(X_{1}(t), X_{2}(t), \ldots, X_{m}(t)\right)$.

The exact form of $\Phi$ depends on the mapping construction technique employed. We tested four methods here, one linear and three nonlinear. Their brief descriptions are in Sects. 2.2.1 (multiple linear regression), 2.2.2 (local linear models), 2.2.4 (MLP neural networks) and 2.2.5 (RBF neural networks). The respective forms of $\Phi$ are represented by Eqs. (4), (5), (6) and (8).

\subsubsection{Multiple linear regression}

Linear methods still represent the most frequently used tool of time series analysis. They are usually less complicated than their nonlinear counterparts, with lower demands regarding computational power, and, unlike nonlinear methods, without many parameters to be determined prior to their application. We used multiple linear regression (MLR) here as a typical representative of linear techniques. The mapping had the form

$$
x^{\operatorname{PRED}}(t)=v_{0}+\sum_{i=1}^{m} v_{i} X_{i}(t),
$$

where $v_{i}, i=0, \ldots, m$, are the regression coefficients.

\subsubsection{Method of local linear models}

Origin of the method of local models dates back to the second half of the eighties and it is associated with research focused on the problems of chaotic dynamics, strange attractors and phase space reconstruction. The method was shown to be suitable for the prediction of low-dimensional chaotic systems (Farmer and Sidorowich, 1987), as well as simple physical (e.g. Sauer, 1993) or biological (e.g. Sugihara and May, 1990) systems. In meteorology, too, its applications have been demonstrated, for example, for cloud coverage forecast (Pérez-Muñuzuri and Gelpi, 2000) or rainfall prediction (Sivakumar et al., 2000). A detailed description of the method and more examples of its application can be found in the monographs by Abarbanel (1996) or Kantz and Schreiber (1997).

Since successful phase space reconstruction enables characterization of the system's state by an $m$-dimensional vector like Eq. (1), it is also possible to quantify the similarity of different states, typically by computing the Euclidian distance of their respective vectors $\boldsymbol{y}$. In order to construct a mapping approximating the time evolution from time $t$ or spatial relation between different variables in time $t$, a certain number $N$ of states $\boldsymbol{y}(t, j), j=1, \ldots, N$, is found in the history of the system as states with the smallest distance to $\boldsymbol{y}(t)$. From the relation between such states and the corresponding values $x(t, j)$ of the predictand, a mapping can be constructed which approximates some local neighbourhood of $\boldsymbol{y}(t)$ in the phase space by a linear model:

$x^{P R E D}(t)=v_{0}(t)+\sum_{i=1}^{m} v_{i}(t) X_{i}(t)$.

Note that, unlike in Eq. (4), coefficients $v_{i}(t)$ are not timeinvariant and they need to be computed separately for each time $t$. Computation of $m+1$ coefficients $v_{i}(t)$ from $N$ pairs of $\boldsymbol{y}(t, j)$ and $x(t, j)$ is a linear regression problem, solvable in the least-squares sense. It should be mentioned that using a fixed value of the number of nearest neighbors $N$ is not the only possible way of defining local neighborhood in the phase space. It is also possible to work with the directly specified size of the neighborhood (e.g. Hegger et al., 1999), or to pick an individual value of $N$ for every $t$, as done by Jayawardena et al. (2002). Here, however, all local models were realized using a constant $N$ for the entire analyzed series.

\subsubsection{Artificial neural networks}

Neural networks (NNs) have become very popular in various scientific areas as a convenient tool for many practical tasks, including time series analysis and data processing. Typical artificial neural network is a complex structure, consisting of some number of interconnected, simple signal processing units - artificial neurons (or, shortly, neurons). Neurons typically have several inputs and a single output; the information received by inputs is processed by the neuron and the outcome is then transmitted to its neighbors. For data processing tasks, so-called feedforward NNs are the ones most frequently applied. A typical feedforward NN consists of several layers of neurons - one, called the input layer, which receives inputs, then one or more hidden layers of signal-processing neurons, and finally, an output layer in which the results are computed to their final form. The output layer can comprise of one or more neurons (producing one or more output values simultaneously), but we only used single-output networks here. Two different types of feedforward networks were studied in this paper: MultiLayer Perceptrons (MLPs) and Radial Basis Function (RBF) networks. For more information on NNs, see, e.g. monographs by Haykin (1999) or Principe et al. (2000).

\subsubsection{Multilayer perceptron neural networks}

MultiLayer Perceptrons (MLPs) are by far the best known and most frequently used design of neural networks - to such an extent that they are sometimes (incorrectly) viewed as being equivalent to NNs generally. The operation performed by neurons in the hidden layer of MLP is a weighted summation. For MLP with one hidden layer and a single neuron in the output layer, the transfer function can be expressed as

$$
x^{\operatorname{PRED}}(t)=v_{0}+\sum_{i=1}^{M} v_{i} f\left(w_{0 i}+\sum_{j=1}^{m} w_{j i} X_{j}(t)\right),
$$




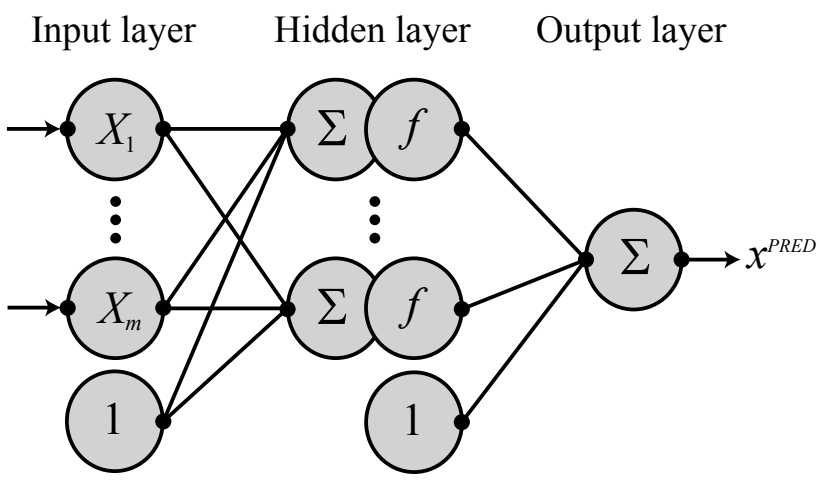

Fig. 2. The schematic structure of multilayer perceptron neural network.

where $w_{j i}$ is the weight of the connection between the $i$-th neuron in the hidden layer and the $j$-th neuron in the input layer, $v_{i}$ is the weight of the connection between the $i$-th neuron in the hidden layer and the neuron in the output layer, and $M$ is the number of neurons in the hidden layer (structure of a MLP network is schematically shown in Fig. 2). Function $f(x)$, called the activation function, is generally nonlinear. We used the logistic function,

$f(x)=\frac{1}{1+\exp (-x)}$.

Apparently, the character of the mapping in Eq. (6) is given by the values of the weights $v_{i}$ and $w_{j i}$, which have to be determined before the network can be used. Values of weights are computed using examples of input-output pairs from some training set (supervised learning). The basic learning technique, backpropagation of errors, is an iterative procedure using values of errors for the training cases to calculate the weights adjustments. The backpropagation technique exists in several variants, differing in their implementation complexity, needed learning time, and tendency to be trapped in a local minimum of the error function instead of reaching the global minimum. We have used the quasi-Newton method for MLPs training (e.g. Haykin, 1999), with weights initialized to uniformly distributed random values.

\subsubsection{Radial basis function neural networks}

In many aspects similar to MLPs, radial basis function neural networks (RBF NNs - Fig. 3) are feedforward networks with one hidden layer and one or more neurons in the output layer. There are two principal differences between MLPs and RBF networks. First, instead of the weighted summation of the inputs, performed by the neurons in the hidden layer of MLPs, RBF networks employ radial basis functions, usually the Gaussian ones. The transfer function can be expressed as

$$
x^{\operatorname{PRED}}(t)=v_{0}+\sum_{i=1}^{M} v_{i} \exp \left(-\frac{\left\|\boldsymbol{y}(t)-\boldsymbol{c}_{i}\right\|^{2}}{2 \sigma_{i}^{2}}\right),
$$

Input layer Hidden layer Output layer

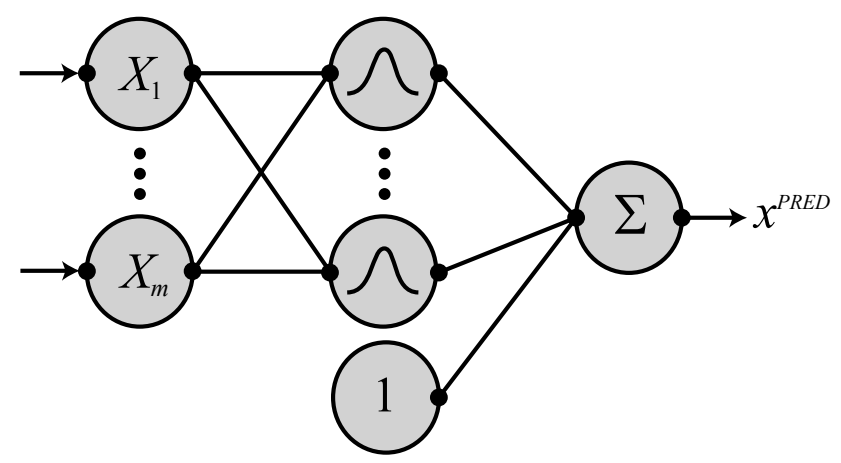

Fig. 3. The schematic structure of radial basis function neural network.

where $c_{i}$ is the position of the centre of the $i$-th radial basis function (assigned to the $i$-th neuron in the hidden layer, $i=1$, $\ldots, M), \sigma_{i}$ characterizes the width of the $i$-th RBF, and the meaning of $v_{i}$ and $M$ is the same as in Eq. (6).

The second major difference between RBF and MLP neural networks is in the learning algorithm. While weights in MLPs are determined in some sort of iterative learning procedure, training of RBF NNs can be done in two separate phases. First, positions and shapes of the radial basis functions are specified (i.e. $c_{i}$ and $\sigma_{i}, i=1, \ldots, M$ ). The simplest way to do this is to take $M$ randomly chosen times $t_{1}, t_{2}, \ldots$, $t_{M}$ from the training set and use the corresponding vectors in the input space as centres of RBFs, $\boldsymbol{c}_{i}=\boldsymbol{y}\left(t_{i}\right), i=1, \ldots, M$. A more sophisticated way of setting $\boldsymbol{c}_{i}$, k-means clustering (MacQueen, 1967; Haykin, 1999), did not notably improve the results in our case. Values of $\sigma_{i}$ can be set individually for each neuron, but in praxis a single value is often used, $\sigma_{i}=\sigma, i=1, \ldots, M$, which we followed. As soon as the positions of RBF centres and sigma are set, finding the values of weights $v_{i}, i=0, \ldots, M$, is a linear problem which can be easily solved using the least-squares approach.

\subsection{Computation settings}

When testing a time series analysis method, it is important to construct the mapping from one part of the data set, and then to test its performance on an independent interval. For neural networks, the former set is usually referred to as a training set while the latter is called a testing set. We use the same convention for LLM and MLR methods, too.

All utilized nonlinear methods here need one or more parameters to be determined before they can be applied (number of nearest neighbors $N$ for LLM method, number of hidden neurons $M$ for both types of neural networks, width of radial functions $\sigma$ for RBF networks). The parameters were chosen to give the lowest RMSE for the testing set (a range of parameter values was tested and the best performing one was then used for the actual computation). RMSE dependence on the above mentioned parameters typically had a broad, flat minimum and so it was relatively easy to pick 
their optimal values. An exception to this rule was observed for the number of hidden neurons in MLPs, when the dependence of RMSE on $M$ exhibited notable fluctuations, due to the sensitivity of the results to the initial values of the network's weights. This was compensated for by repeated training (see below), but even then the error curve was visibly less smooth than for RMSE as a function of $N$ or RBF NNs' $M$.

Optimization of the parameters was done for the entire year as a whole, not separately for individual seasons. For different tasks, the number of nearest neighbors for the LLM method ranged between 200 and 700. As for the MLP neural networks, the best results were typically obtained with the number of hidden neurons 8 to 12 , but sometimes as high as 20. In the case of RBF NNs, the optimal number of hidden units was much higher, usually between 200 and 400. The width of radial functions was set to $\sigma=3.2$ (very little sensitivity of the results to its value was observed, so we kept it fixed for all tasks). Training of neural networks, both MLP and RBF, was performed 5 times from random initial weights (or RBF centres' positions, respectively) and the presented results are an average of this five-member ensemble. In retrospect, repeated learning was probably not necessary for the RBF networks, since variance of the results within the ensemble was quite low. In the case of MLPs, a single realization could profoundly misrepresent the performance of the method, so using an entire ensemble was important to reduce the effect of sensitivity to the initial values of weights. Input values for all neural networks were normalized to range $[0,1]$ by subtracting the minimum of the series and then dividing the value with the series' max-min range. MLP networks were trained for 1500 epochs by the quasi-Newton method.

An important part of the input data is information about the season of the year. In order to introduce this information to the computations, application of the MLR and LLM methods was done separately for each season. For NNs, we did perform the training for the entire training set as a whole, and information about the season was introduced by four extra neurons in the input layer, each of which was assigned to one season (the effective dimension of the input space was therefore $m=18$ for all neural networks). These neurons' activations were equal to 1 for the season they controlled, and 0 otherwise (i.e. just one of these four neurons was active for a given time - the one assigned to the corresponding season). Another possibility of introducing information about the season would be using sine and cosine of the Julian day (as done by Trigo and Palutikof, 1999). The usual climatological definition of the seasons was utilized:

$$
\begin{aligned}
& \text { Winter = December }+ \text { January }+ \text { February = DJF, } \\
& \text { Spring = March }+ \text { April }+ \text { May = MAM, } \\
& \text { Summer }=\text { June }+ \text { July }+ \text { August } \quad=\text { JJA and } \\
& \text { Autumn }=\text { September }+ \text { October }+ \text { November }=\text { SON } \text {. }
\end{aligned}
$$

\section{Data}

The first data set utilized was the NCEP/NCAR reanalysis by Kistler et al. (2001), obtained from the page of NOAA-
CIRES CDC at http://www.cdc.noaa.gov. It is available for the years from 1948 on, and it covers an entire world with reanalysis of several meteorological variables in the regular $2.5^{\circ} \times 2.5^{\circ}$ grid, including data for various pressure levels. Series of mean daily temperature at the level $1000 \mathrm{hPa}$ $\left(T_{1000}\right)$ were used here, as well as series of mean sea level pressure (MSLP) and series of geopotential height of the $500 \mathrm{hPa}$ level $\left(h_{500}\right)$.

As for directly measured meteorological series, perhaps the largest publicly available data set of European temperature, precipitation and pressure measurements was collected by Klein Tank et al. (2002) and it is obtainable from the Internet page of the European Climate Assessment and Dataset (ECA\&D - http://eca.knmi.nl/). Measurements from many different sources were assembled by the authors, so series of various quality and length are part of ECA\&D, and many of them contain missing values. We used series of daily mean, minimum and maximum temperature from 25 European stations - see Table 1 for their list. Many more series can be obtained from ECA\&D - these $3 \times 25$ were selected in order to cover most of Europe with series available for the years 1961 to 2000, or ending not too long before the year 2000, and containing as little missing values as possible.

\section{Results}

\subsection{Prediction}

Forecast of future weather is what most people view as a fundamental purpose of meteorology's existence. And although today's weather forecasts are made almost exclusively by means of numerical models, there are many supplementary tasks for which time series analysis can be more suitable, for example, because the available data do not allow for use of a generally demanding NWF model, because of unsuitable spatial or temporal scale or due to a lack of available computing capacity. It was shown that nonlinear methods can be applied for tasks like prediction of road temperatures (Shao, 1998), precipitation forecasting (Waelbroeck et al., 1994; Hall et al., 1999; Sivakumar et al., 2000), or forecast of cloud cover (Pérez-Muñuzuri and Gelpi, 2000), although in some cases nonlinear methods do not seem to be more suitable than the linear ones, as demonstrated, for example, by Tang et al. (2000) for Central Pacific SST forecast.

The gridded NCEP/NCAR reanalysis data set represents a suitable basis for study of spatial distribution of the predictive potential of nonlinear methods. First, we performed the prediction of the $T_{1000}$ series one day ahead by the LLM and MLR methods for every grid point in the area between $65^{\circ} \mathrm{N}, 25^{\circ} \mathrm{W}$ and $25^{\circ} \mathrm{N}, 45^{\circ} \mathrm{E}$, i.e. for 493 points in total. Years 1961-1990 were used as the training set, while the testing set consisted of the years 1991-2000. The structure of the predictor space is demonstrated in Fig. 1a for the grid point $50^{\circ} \mathrm{N}, 15^{\circ} \mathrm{E}$ (the pattern was moved to be centered on the location of the predicted series). Unlike for all the other computations in this paper, a fixed number of nearest 
Table 1. A list of stations used for downscaling tests. Asterisk $(*)$ marks the stations for which at least one of the series of daily mean, minimum or maximum temperature did not cover the entire period 1961-2000.

\begin{tabular}{|c|c|c|c|c|c|}
\hline Station & Country & Latitude & Longitude & $\begin{array}{l}\text { Alt. } \\
(\mathrm{m})\end{array}$ & $\begin{array}{l}\text { NCEP/NCAR } \\
\text { nearest grid point }\end{array}$ \\
\hline Beograd* & Yugoslavia & $44^{\circ} 48^{\prime} \mathrm{N}$ & $20^{\circ} 28^{\prime} \mathrm{E}$ & 132 & $45^{\circ} 00^{\prime} \mathrm{N}, 20^{\circ} 00^{\prime} \mathrm{E}$ \\
\hline Berlin & Germany & $52^{\circ} 27^{\prime} \mathrm{N}$ & $13^{\circ} 18^{\prime} \mathrm{E}$ & 55 & $52^{\circ} 30^{\prime} \mathrm{N}, 12^{\circ} 30^{\prime} \mathrm{E}$ \\
\hline Bordeaux & France & $44^{\circ} 50^{\prime} \mathrm{N}$ & $00^{\circ} 41^{\prime} \mathrm{W}$ & 47 & $45^{\circ} 00^{\prime} \mathrm{N}, 00^{\circ} 00^{\prime}$ \\
\hline Bologna & Italy & $44^{\circ} 29^{\prime} \mathrm{N}$ & $11^{\circ} 15^{\prime} \mathrm{E}$ & 60 & $45^{\circ} 00^{\prime} \mathrm{N}, 10^{\circ} 00^{\prime} \mathrm{E}$ \\
\hline Bremen & Germany & $53^{\circ} 03^{\prime} \mathrm{N}$ & $08^{\circ} 47^{\prime} \mathrm{E}$ & 4 & $52^{\circ} 30^{\prime} \mathrm{N}, 10^{\circ} 00^{\prime} \mathrm{E}$ \\
\hline Brindisi* & Italy & $40^{\circ} 38^{\prime} \mathrm{N}$ & $17^{\circ} 56^{\prime} \mathrm{E}$ & 10 & $40^{\circ} 00^{\prime} \mathrm{N}, 17^{\circ} 30^{\prime} \mathrm{E}$ \\
\hline Eskdalemuir & UK & $55^{\circ} 19^{\prime} \mathrm{N}$ & $03^{\circ} 12^{\prime} \mathrm{W}$ & 242 & $55^{\circ} 00^{\prime} \mathrm{N}, 02^{\circ} 30^{\prime} \mathrm{W}$ \\
\hline Helsinki & Finland & $60^{\circ} 10^{\prime} \mathrm{N}$ & $24^{\circ} 57^{\prime} \mathrm{E}$ & 4 & $60^{\circ} 00^{\prime} \mathrm{N}, 25^{\circ} 00^{\prime} \mathrm{E}$ \\
\hline Heraklion* & Greece & $35^{\circ} 20^{\prime} \mathrm{N}$ & $25^{\circ} 11^{\prime} \mathrm{E}$ & 39 & $35^{\circ} 00^{\prime} \mathrm{N}, 25^{\circ} 00^{\prime} \mathrm{E}$ \\
\hline Hurbanovo & Slovakia & $47^{\circ} 53^{\prime} \mathrm{N}$ & $18^{\circ} 12^{\prime} \mathrm{E}$ & 115 & $47^{\circ} 30^{\prime} \mathrm{N}, 17^{\circ} 30^{\prime} \mathrm{E}$ \\
\hline Karlsruhe & Germany & $49^{\circ} 01^{\prime} \mathrm{N}$ & $08^{\circ} 23^{\prime} \mathrm{E}$ & 114 & $50^{\circ} 00^{\prime} \mathrm{N}, 07^{\circ} 30^{\prime} \mathrm{E}$ \\
\hline Kremsmuenster* & Austria & $48^{\circ} 03^{\prime} \mathrm{N}$ & $14^{\circ} 08^{\prime} \mathrm{E}$ & 383 & $47^{\circ} 30^{\prime} \mathrm{N}, 15^{\circ} 00^{\prime} \mathrm{E}$ \\
\hline Larissa* & Greece & ${ }^{\circ} 39^{\prime} \mathrm{N}$ & $22^{\circ} 27^{\prime} \mathrm{E}$ & 74 & $40^{\circ} 00^{\prime} \mathrm{N}, 22^{\circ} 30^{\prime} \mathrm{E}$ \\
\hline St. Petersburg* & Russia & $59^{\circ} 58^{\prime} \mathrm{N}$ & $30^{\circ} 18^{\prime} \mathrm{E}$ & 6 & $60^{\circ} 00^{\prime} \mathrm{N}, 30^{\circ} 00^{\prime} \mathrm{E}$ \\
\hline Linkoeping & & $58^{\circ} 24^{\prime} \mathrm{N}$ & $15^{\circ} 32^{\prime} \mathrm{E}$ & 93 & $57^{\circ} 30^{\prime} \mathrm{N}, 15^{\circ} 00^{\prime} \mathrm{E}$ \\
\hline Malaga & Spain & $36^{\circ} 40^{\prime} \mathrm{N}$ & $04^{\circ} 29^{\prime} \mathrm{W}$ & 7 & $37^{\circ} 30^{\prime} \mathrm{N}, 05^{\circ} 00^{\prime} \mathrm{W}$ \\
\hline Marseille & & $43^{\circ} 18^{\prime} \mathrm{N}$ & $05^{\circ} 24^{\prime} \mathrm{E}$ & 75 & $42^{\circ} 30^{\prime} \mathrm{N}, 05^{\circ} 00^{\prime} \mathrm{E}$ \\
\hline Moskou* & Russia & $55^{\circ} 50^{\prime} \mathrm{N}$ & $37^{\circ} 37^{\prime} \mathrm{E}$ & 156 & $55^{\circ} 00^{\prime} \mathrm{N}, 37^{\circ} 30^{\prime} \mathrm{E}$ \\
\hline Oslo Blindern & Norway & $59^{\circ} 57^{\prime} \mathrm{N}$ & $10^{\circ} 43^{\prime} \mathrm{E}$ & 94 & $60^{\circ} 00^{\prime} \mathrm{N}, 10^{\circ} 00^{\prime} \mathrm{E}$ \\
\hline Oxford & UK & $51^{\circ} 46^{\prime} \mathrm{N}$ & $01^{\circ} 16^{\prime} \mathrm{W}$ & 63 & $52^{\circ} 30^{\prime} \mathrm{N}, 02^{\circ} 30^{\prime} \mathrm{W}$ \\
\hline Paris & France & $48^{\circ} 49^{\prime} \mathrm{N}$ & $02^{\circ} 20^{\prime} \mathrm{E}$ & 75 & $50^{\circ} 00^{\prime} \mathrm{N}, 02^{\circ} 30^{\prime} \mathrm{E}$ \\
\hline Praha & Czech Rep. & $50^{\circ} 05^{\prime} \mathrm{N}$ & $14^{\circ} 25^{\prime} \mathrm{E}$ & 191 & $50^{\circ} 00^{\prime} \mathrm{N}, 15^{\circ} 00^{\prime} \mathrm{E}$ \\
\hline Tortosa & Spain & $40^{\circ} 49^{\prime} \mathrm{N}$ & $00^{\circ} 29^{\prime} \mathrm{E}$ & 48 & $40^{\circ} 00^{\prime} \mathrm{N}, 00^{\circ} 00^{\prime}$ \\
\hline Utsira Fyr & Norway & $59^{\circ} 18^{\prime} \mathrm{N}$ & $04^{\circ} 53^{\prime} \mathrm{E}$ & 55 & $60^{\circ} 00^{\prime} \mathrm{N}, 05^{\circ} 00^{\prime} \mathrm{E}$ \\
\hline Valentia Observatory* & Ireland & $51^{\circ} 56^{\prime} \mathrm{N}$ & $10^{\circ} 13^{\prime} \mathrm{W}$ & 9 & $52^{\circ} 30^{\prime} \mathrm{N}, 10^{\circ} 00^{\prime} \mathrm{W}$ \\
\hline
\end{tabular}

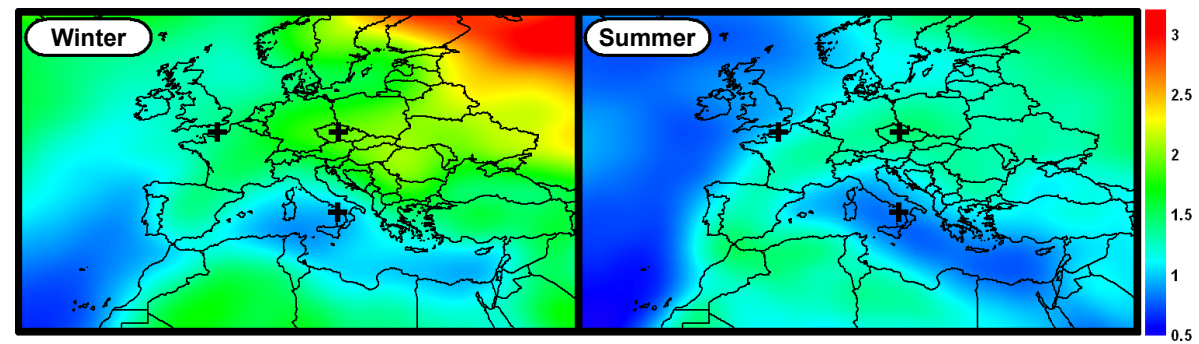

Fig. 4. RMSE $\left({ }^{\circ} \mathrm{C}\right)$ of 1 -day ahead prediction of temperature at the $1000 \mathrm{hPa}$ level $\left(T_{1000}\right)$ by LLM method. Three black crosses mark the positions of the grid points from Table 2 .

neighbors was used for all grid points, $N=250$. The results are shown in Fig. 4 (RMSE for LLM method) and 5 (RMSE for LLM method, divided with RMSE for MLR, which we will call effective nonlinearity, since it quantifies an improvement which can be gained from replacing linear MLR with the nonlinear method of local models).

The lowest values of RMSE were detected over the Mediterranean Sea and Atlantic Ocean, and, in summer, in the Near East. Errors in summer were generally lower than in winter. The absolute values of the errors coincide well with values of average interdiurnal temperature change, i.e. errors were higher in the areas with higher temperature variability. When the spatial structure of effective nonlinearity was analyzed, the observed pattern was more complex. In winter, most of continental Europe appears to be a region of increased nonlinearity, with the highest differences between RMSE for the LLM and MLR methods in Western Europe and Russia. Nonlinearity seems to be rather weak in the maritime areas, as well as in Northern Africa and the Near East. In summer, nonlinearity is weaker than in winter in most areas, and there is a clear contrast between the Mediterranean area (very weak nonlinearity, except for Tunisia and Northern Libya) and the rest of Europe (rather stronger nonlinearity, especially in Northern France, Belgium and Netherlands). This observed pattern bears an interesting resemblance to the positions of the climatic zones, as nonlinearity 
Table 2. RMSE $\left({ }^{\circ} \mathrm{C}\right)$ of 1-day ahead prediction of NCEP/NCAR $T_{1000}$ series for three different grid points. The values in bold indicate that the nonlinear method gave better results than multiple linear regression, according to the paired Wilcoxon test at the $95 \%$ confidence level. The values in the first row show RMSE of persistent prediction.

\begin{tabular}{llllllllllllll}
\hline & \multicolumn{3}{l}{$\mathbf{5 0}^{\circ} \mathbf{N}, \mathbf{0}^{\circ} \mathbf{E}$} & \multicolumn{4}{c}{$\mathbf{5 0}^{\circ} \mathbf{N}, \mathbf{1 5}^{\circ} \mathbf{E}$} & \multicolumn{5}{c}{$\mathbf{4 0}^{\circ} \mathbf{N}, \mathbf{1 5}^{\circ} \mathbf{E}$} \\
& DJF & MAM & JJA & SON & DJF & MAM & JJA & SON & DJF & MAM & JJA & SON \\
\hline Pers. & 2.12 & 1.8 & 1.73 & 1.74 & 2.73 & 2.41 & 2.31 & 2.31 & 1.42 & 1.15 & 0.99 & 1.23 \\
MLR & 1.75 & 1.48 & 1.3 & 1.5 & 2.3 & 1.9 & 1.65 & 1.86 & 1.11 & 0.98 & 0.83 & 0.99 \\
& & & & & & & & & & & & & \\
LLM & $\mathbf{1 . 4 1}$ & $\mathbf{1 . 2 8}$ & $\mathbf{1 . 0 7}$ & $\mathbf{1 . 2 1}$ & $\mathbf{1 . 9 3}$ & $\mathbf{1 . 5 7}$ & $\mathbf{1 . 4 4}$ & $\mathbf{1 . 5 4}$ & $\mathbf{0 . 9 6}$ & $\mathbf{0 . 8 8}$ & 0.83 & $\mathbf{0 . 8 8}$ \\
MLP & $\mathbf{1 . 5 2}$ & $\mathbf{1 . 3 5}$ & 1.18 & $\mathbf{1 . 2 4}$ & $\mathbf{1 . 9}$ & $\mathbf{1 . 5 4}$ & $\mathbf{1 . 4 6}$ & $\mathbf{1 . 5 7}$ & $\mathbf{0 . 9 8}$ & 0.92 & 0.81 & $\mathbf{0 . 8 9}$ \\
RBF & $\mathbf{1 . 3 9}$ & $\mathbf{1 . 2 6}$ & $\mathbf{1 . 0 7}$ & $\mathbf{1 . 1 5}$ & $\mathbf{1 . 8 7}$ & $\mathbf{1 . 5 2}$ & $\mathbf{1 . 3 8}$ & $\mathbf{1 . 5 4}$ & $\mathbf{0 . 9 5}$ & $\mathbf{0 . 8 7}$ & $\mathbf{0 . 7 9}$ & $\mathbf{0 . 8 8}$ \\
\hline
\end{tabular}

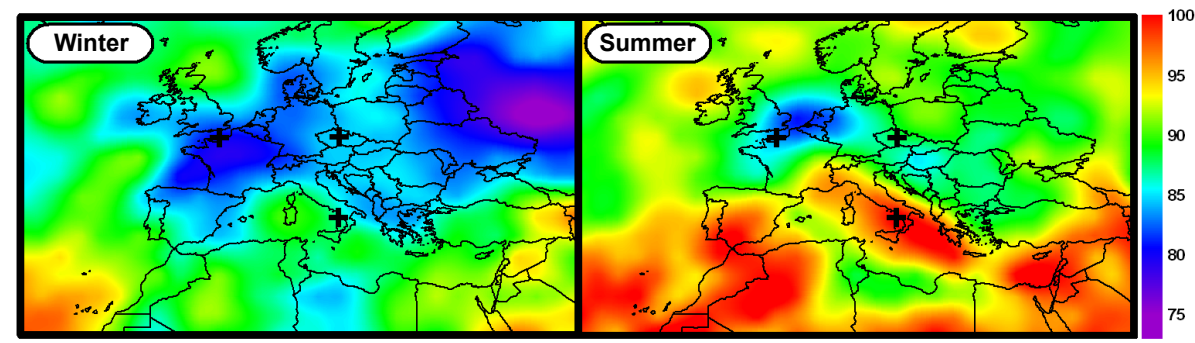

Fig. 5. Effective nonlinearity for 1-day ahead prediction of temperature at the $1000 \mathrm{hPa}$ level $\left(T_{1000}\right)$, i.e. RMSE for LLM method expressed in \% of RMSE for multiple linear regression (lower values indicate stronger nonlinearity). Three black crosses mark the positions of the grid points from Table 2 .

seems to be stronger in the temperate zone and weaker in the subtropical and tropical areas (various climate classifications can be found in Essenwanger, 2001).

There is a comparison of 1-day ahead prediction results in Table 2 for all tested methods and all seasons of the year for three selected NCEP/NCAR grid points: $50^{\circ} \mathrm{N}, 0^{\circ} \mathrm{E}$ (a grid point which exhibited strong effective nonlinearity in both summer and winter), $50^{\circ} \mathrm{N}, 15^{\circ} \mathrm{E}$ (medium nonlinearity in both seasons) and $40^{\circ} \mathrm{N}, 15^{\circ} \mathrm{E}$ (medium nonlinearity in winter, very weak in summer). Positions of the respective grid points are indicated by crosses in Figs. 4 and 5. Aside from comparing RMSEs, differences between individual methods were ascertained using the paired Wilcoxon test (e.g. Wilks, 1995), applied at the absolute values of daily errors. The significance of the differences between errors from the MLR and nonlinear methods is indicated in Table 2 - values in bold signal that the nonlinear method was better than MLR at the 95\% level of confidence. RBF NN typically gave the best results of all three nonlinear methods in terms of RMSE, but the difference of error medians was not significant, according to the Wilcoxon test, in most of the tested cases. On the other hand, nonlinear methods, especially LLM and RBF NNs, typically gave better results than MLR, both with respect to RMSE and to the significance of the difference of daily errors.

Visually, there was just little difference between the series of predictions from all four tested methods. Superiority of nonlinear techniques in the geographic areas with strong ef-

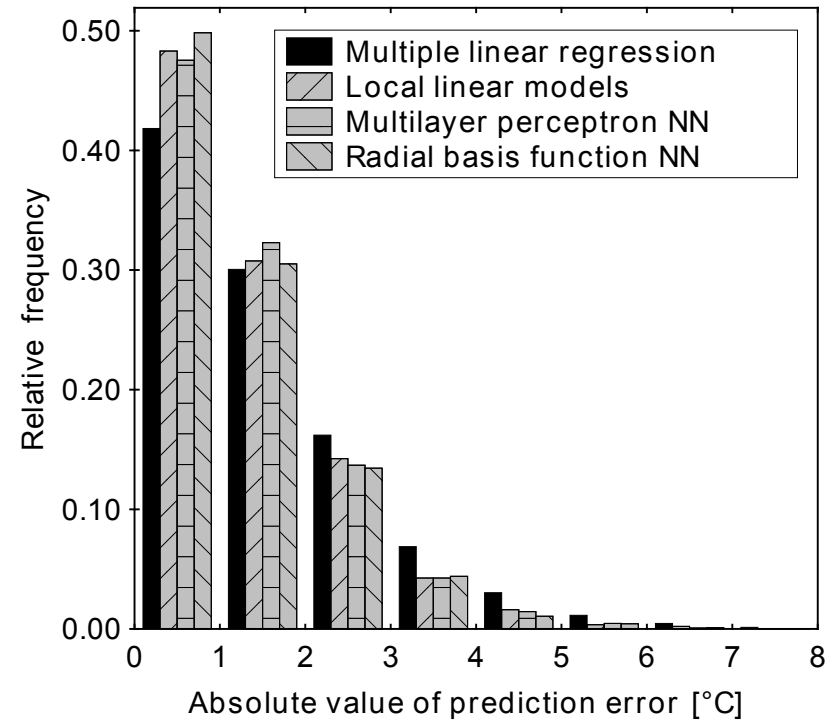

Fig. 6. Histogram of the absolute values of the prediction errors for 1-day ahead prediction of NCEP/NCAR $T_{1000}$ series at $50^{\circ} \mathrm{N}$, $15^{\circ} \mathrm{E}$ (whole year).

fective nonlinearity was, however, clearly visible in the distribution of errors, with residuals close to zero being relatively more frequent for nonlinear methods than for MLR - see example in Fig. 6. What all methods had in common was a certain tendency to underestimate high values of temperature and overestimate the low ones, thus actually 
Table 3. RMSE $\left({ }^{\circ} \mathrm{C}\right)$ of NCEP/NCAR reanalysis downscaling for four European stations. The values in bold indicate that the nonlinear method gave better results than multiple linear regression, according to the Wilcoxon test at the $95 \%$ confidence level. Underline indicates that the method performed better than all the other three, according to the Wilcoxon test. The values in the first row for each station show RMSE obtained by means of mean climatology.

\begin{tabular}{|c|c|c|c|c|c|c|c|c|c|c|c|c|c|}
\hline & & \multicolumn{4}{|c|}{ Mean temperature } & \multicolumn{4}{|c|}{ Min. temperature } & \multicolumn{4}{|c|}{ Max. temperature } \\
\hline & & DJF & MÄM & JJA & SON & DJF & MAM & JJA & SON & DJF & MAM & JJA & SON \\
\hline \multirow{4}{*}{ Praha } & Clim. & 4.68 & 3.96 & 3.44 & 3.53 & 4.75 & 3.45 & 2.71 & 3.50 & 4.81 & 4.71 & 4.31 & 4.07 \\
\hline & LLM & 1.73 & 1.26 & 1.17 & 1.28 & 2.23 & 1.72 & 1.44 & 1.74 & $\underline{1.67}$ & 1.66 & 1.52 & 1.69 \\
\hline & MLP & 1.77 & 1.24 & 1.20 & 1.34 & 2.16 & 1.70 & 1.41 & 1.75 & $\overline{1.72}$ & 1.63 & 1.52 & 1.68 \\
\hline & $\mathbf{R B F}$ & 1.75 & $\underline{1.22}$ & 1.21 & 1.29 & 2.18 & 1.70 & 1.42 & 1.72 & 1.73 & 1.63 & 1.52 & 1.68 \\
\hline \multirow{4}{*}{ Oslo } & MLR & 2.44 & 1.64 & 1.33 & 1.58 & 2.96 & 2.00 & 1.71 & 2.19 & 2.54 & 2.38 & 1.92 & 1.97 \\
\hline & LLM & 2.12 & 1.65 & $\underline{1.30}$ & 1.49 & 2.69 & 1.96 & 1.72 & 2.09 & $\underline{2.27}$ & 2.34 & 1.88 & 1.86 \\
\hline & MLP & 2.13 & 1.57 & $\overline{1.33}$ & 1.50 & 2.69 & 1.83 & 1.67 & 2.06 & $\overline{2.28}$ & 2.31 & 1.89 & 1.77 \\
\hline & $\mathbf{R B F}$ & 2.10 & 1.59 & 1.32 & 1.45 & 2.67 & 1.84 & 1.67 & 2.09 & 2.26 & 2.29 & 1.87 & $\underline{1.74}$ \\
\hline Oxford & Clim. & 3.46 & 2.86 & 2.46 & 2.83 & 3.78 & 3.14 & 2.45 & 3.44 & 3.66 & 3.37 & 3.40 & 2.93 \\
\hline \multirow{5}{*}{ Bordeaux } & Clim. & 3.75 & 3.13 & 2.97 & 3.25 & 4.25 & 3.16 & 2.72 & 3.63 & 3.98 & 4.25 & 4.15 & 3.86 \\
\hline & MLR & 1.75 & 1.47 & 1.45 & 1.50 & 2.50 & 2.38 & 2.18 & 2.37 & 2.26 & 2.05 & 1.87 & 1.87 \\
\hline & LLM & 1.69 & 1.42 & 1.45 & $\underline{1.44}$ & 2.40 & $\underline{2.22}$ & 2.11 & 2.21 & 2.18 & 2.00 & 1.88 & 1.85 \\
\hline & MLP & 1.72 & 1.44 & 1.40 & $\overline{1.45}$ & 2.39 & $\overline{2.25}$ & 2.07 & 2.19 & 2.19 & 1.97 & 1.85 & 1.80 \\
\hline & RBF & 1.66 & 1.43 & 1.40 & 1.45 & 2.38 & 2.26 & 2.07 & 2.22 & 2.16 & 2.01 & 1.86 & 1.81 \\
\hline
\end{tabular}

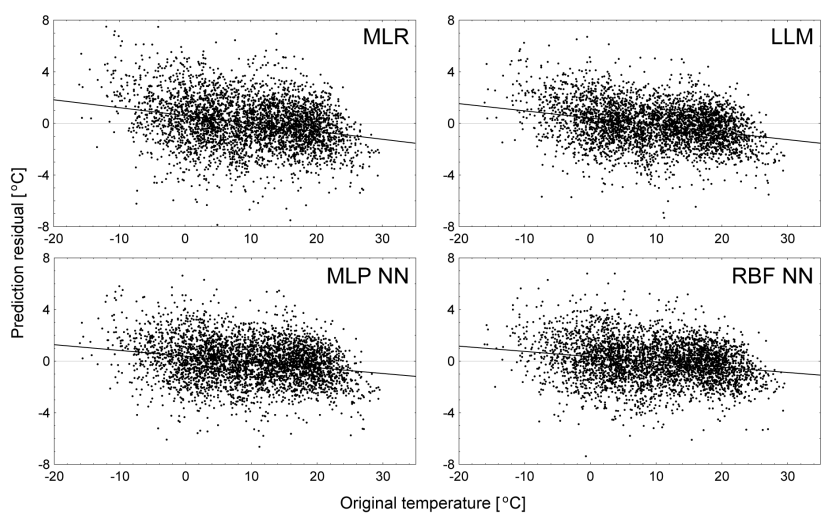

Fig. 7. Plot of the prediction residuals (the predicted temperature minus the original observed temperature) against the original temperature for 1-day ahead prediction of NCEP/NCAR $T_{1000}$ series at $50^{\circ} \mathrm{N}, 15^{\circ} \mathrm{E}$ (whole year). Bold line represents the linear fit of the residuals.

decreasing the variance of the series of predictions compared to the original one. This kind of behavior was observed for all seasons, as well as the year as a whole, and it is demonstrated for the grid point $50^{\circ} \mathrm{N}, 15^{\circ} \mathrm{E}$ in Fig. 7. The problem of a deformed distribution of values is commonly encoun- tered in the context of the application of empirical models in climate research, particularly in statistical downscaling, and various strategies have been proposed for handling it, such as variance inflation or partial randomization (e.g. von Storch, 1999). All results presented here are direct outputs of the transfer functions, without being subject to any form of additional postprocessing.

\subsection{Downscaling}

Statistical downscaling of large-scale data is another common task of meteorological time series analysis, and one in which nonlinear methods are sometimes used. Several studies have been published devoted to downscaling or postprocessing of temperatures by nonlinear methods, mostly MLPs (Trigo and Palutikof, 1999; Schoof and Pryor, 2001; Marzban, 2003; Casaioli et al., 2003) or neural networks based on RBF functions (Weichert and Bürger, 1998). Here, downscaling of the gridded large-scale data was done for the predictand series of daily mean, minimum and maximum temperatures. NCEP/NCAR reanalysis series were used as predictors. The pattern of predictors (Fig. 1b) was centered on the NCEP/NCAR grid point closest to the respective station (see the last column of Table 1). Years 1961-1990 were used as the training set, and years 1991-2000 as the testing set. In some cases, the testing set was shorter than 10 years 


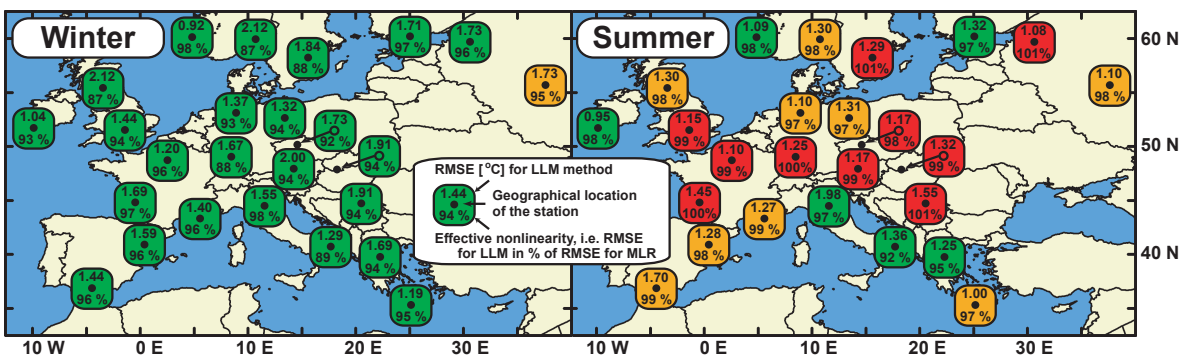

Fig. 8. Results of NCEP/NCAR reanalysis downscaling by LLM and MLR methods for 25 European stations - daily mean temperature. Color of the station's background shows the result of the test of the hypothesis that the absolute values of daily errors were the same for both methods, against the alternative that errors were smaller for LLM - red means that the hypothesis was not rejected at the level of confidence of $95 \%$, yellow that it was rejected at $95 \%$, but not at $99 \%$, and green means that the difference or errors was significant at the $99 \%$ level of confidence (one-sided paired Wilcoxon test was used).

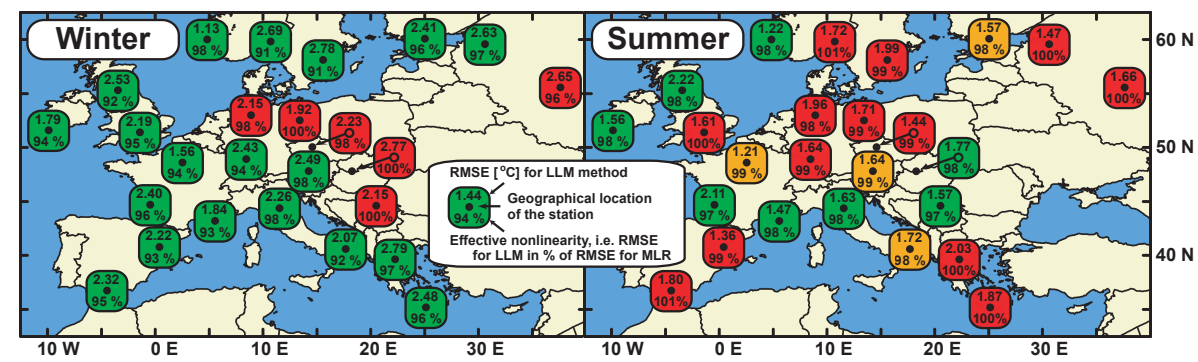

Fig. 9. Same as Fig. 8, for daily minimum temperature.

because the predictand temperature series was not available for the whole interval (such stations are marked with an asterisk in Table 1). Moreover, some of the series contained missing values - in those cases, the respective days were excluded from the computation. The number of missing values was, however, always very small in comparison with the total size of the data set, so their presence should not have caused any major shift in the results.

For all 25 stations from Table 1, results of downscaling by MLR and LLM methods for daily mean (Fig. 8), minimum (Fig. 9) and maximum (Fig. 10) temperature in winter and in summer are presented. Aside from computing the relative differences between RMSE for the LLM and MLR methods, we also tested the statistical significance of the difference of the medians of the absolute values of daily errors for the LLM and MLR methods. The testing was done by the one-sided paired Wilcoxon test, and its outcomes are represented by different colors of the respective station's background in Figs. 8 to 10. Full results for the four stations are shown in Table 3. Situations when nonlinear method gave better results than MLR at the $95 \%$ level of confidence are indicated by bold print. To compare the results to outcomes of a low-skill method, the table also contains RMSE obtained by means of mean climatology (i.e. when monthly mean values of the predictand were used in the role of $x^{P R E D}(t)$ from Eq. (3)).

Nonlinear methods generally performed better than MLR, but there were distinct differences between the seasons most profoundly nonlinear behavior was typical for winter, while in summer, nonlinear techniques seemed to grant just a very small, if any, improvement in the comparison with MLR at most stations. This can be easily seen from Fig. 11, where histograms of the absolute values of downscaling errors are shown for mean daily temperature from Oslo (a station, where the contrast between winter and summer was particularly clear). A tendency was observed in all methods to underestimate high temperatures and overestimate the low ones, as in the case of the forecasts in Sect. 4.1.

Situations when one of the methods was superior to all the others, according to the Wilcoxon test, were rather rare, and they are marked with an underline in Table 3. None of the nonlinear methods could be identified as the best one in all cases, or the majority of the cases. However, when one of the methods was tested as the best performing one, it was usually either the method of local models or the RBF neural network.

Unlike the forecasts studied in Sect. 4.1, results of downscaling were influenced by local conditions of individual stations and by eventual problems with series quality (shorter length of some series, as well as the fact that the series in ECA\&D were collected from many different sources, and they may not be mutually as easily comparable as NCEP/NCAR reanalysis data). Thus, we have not tried to draw any detailed conclusions about the geographical structure of errors or nonlinearity based on the behavior of individual stations. A few points can be made, nonetheless: 


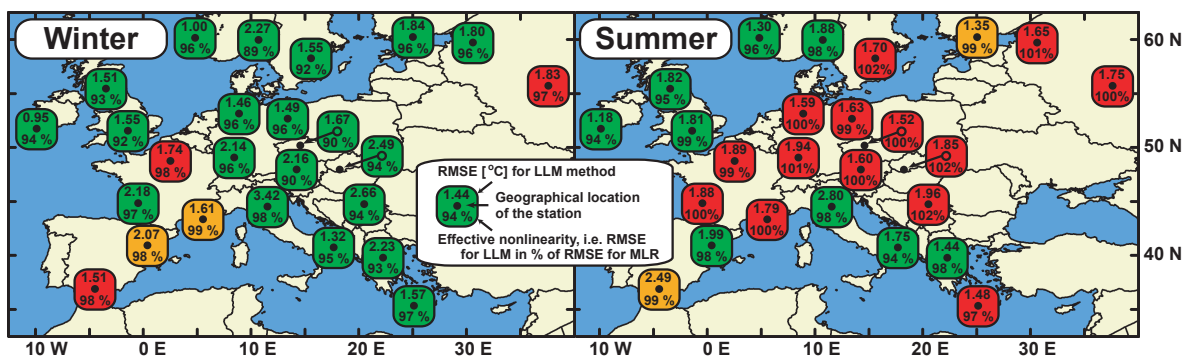

Fig. 10. Same as Fig. 8, for daily maximum temperature.
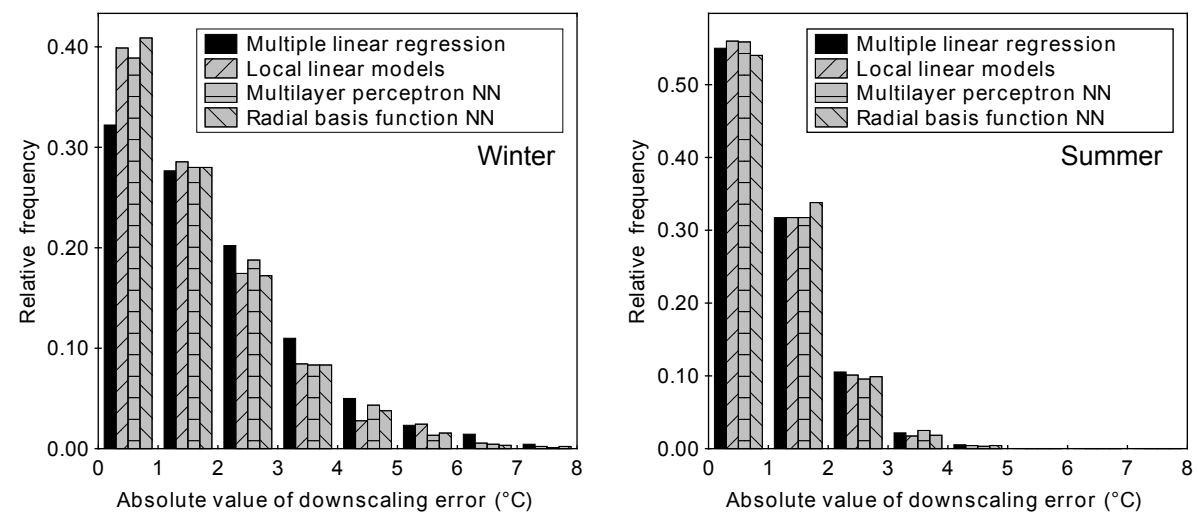

Fig. 11. Histogram of the absolute values of downscaling errors for mean daily temperature from Oslo, Norway, in winter (left) and summer (right).

- Values of RMSE were higher in winter than in summer at most stations, for mean and minimum temperatures; for maximum temperature, almost half of the stations showed higher values of error in summer. The effective nonlinearity was typically stronger in winter than in summer for all three types of temperature. In summer, nonlinear methods even performed a little worse than MLR sometimes, and for many stations, the difference in the medians of errors was not conclusive at the confidence level of $95 \%$.

- Errors were lower for daily mean temperatures than for maximum or minimum ones in most locations. This is not surprising as mean values are already averaged in time, and therefore smoother. The extreme temperatures represent values in a single moment during the day, hence they are more prone to be affected with a noise component of the atmospheric dynamics, and more difficult to determine.

- In Sect. 4.1, we pointed out that nonlinearity in the Mediterranean area seems to be significantly weaker than for the rest of Europe. Here, a similar rule does not apply and there is no clear connection between the geographic position of the station and the degree of exhibited nonlinearity, as even geographically close stations exhibited quite a different behavior in a number of cases.

\section{Discussion}

The presented analyses, carried out for different tasks and various series, do not identify any of the tested nonlinear methods to be definitely superior to the others. It is, however, obvious that in most cases the LLM, MLP NN and RBF NN methods outperformed multiple linear regression. Application of nonlinear methods seems, therefore, to be useful for meteorological time series analysis at synoptic time scales, but the issue remains as to which one of them should be used preferably. Aside from the performance itself, attention should also be paid to the application properties of the contestants. Advantages and drawbacks of individual methods may be summarized as follows:

- Method of local linear models' advantage is that its use need not be preceded by the potentially time-consuming training of weights. On the other hand, the execution itself takes longer than for the already trained NNs, due to the search for nearest neighbors, which needs to be carried out for every processed state. This method is faster than both types of NNs when applied just once for a given setting, as we did in order to obtain the results for Figs. 4 and 5. Its speed also makes estimation of suitable parameters (specifically, $N$ ) by a trial-and-error technique easier.

- MLPs are the most frequently used nonlinear technique of the tested ones, which is an advantage by itself, due 
to support in many existing software applications. Their features are well documented and thoroughly discussed by a number of authors (among others Haykin, 1999; Principe et al., 2000). Perhaps the most severe drawback of MLPs is the possibility that the learning procedure will end in a local minimum of the error function instead of reaching the global one. The seriousness of this problem can be reduced by means of repeated training or by application of a global minimum search technique (such as the one used by Casaioli et al., 2003), but usually at the cost of increased training time. There is also the possibility of overtraining, when the learning procedure runs for too long a time, and the network becomes overoptimized for the description of the cases from the learning set, thus losing its ability to generalize. None of these problems is unsolvable, but they cause the application of MLPs to be potentially tricky, with many nontrivial decisions to be made and the learning process which needs to be supervised.

- RBF NNs do not suffer from the "local minimum trap" problem (at least not their form that we used here), because the supervised optimization of the weights to the output layer can be treated as a linear problem. Certain variations of the results can arise from the random selection of the RBF centers, but these were very small in our case. There is also no need to worry about the network becoming overtrained. Nonetheless, it is still possible to overfit the network in the sense of too many free parameters (when too many hidden neurons are used). Training of RBF NNs is usually faster than that of MLPs (especially considering the need for repeated training of MLPs).

Therefore, the RBF NNs and LLM method can offer better application properties than MLPs in many respects. Considering their relatively easy implementation, we believe that these methods can be recommended as a worthy alternative to MLPs.

The error and nonlinearity maps in Figs. 4, 5 and 8 to 10 offer some insight into the geographic structure of spatiotemporal relations between the atmospheric variables, but they should be interpreted carefully. For prediction (Figs. 4 and 5), the fact that the reanalysis and not measured data was used may be partly responsible for the character of the observed structures. There is also an issue of selection of the input variables - the presented results were obtained for one specific pattern of predictors, and it can be argued that the maps could change if a different one was used. We repeated the tests for various sets of predictors, differing in both type and number of variables used, and although the details of the maps changed, the basic geographic structure of nonlinearity seemed quite robust and unaffected by the modification of the computation settings. There still was an area of distinct nonlinearity over continental Europe, more apparent in winter than in summer, while over the Mediterranean and Northern Africa, nonlinearity was weaker. These results suggest that the nonlinear character of the climate system is strongly reflected in the atmospheric time series in the midlatitudes, and that the relations between them cannot be fully described by linear mappings. In the tropical areas south of Europe, a purely linear description seems more sufficient.

Interpretation of the downscaling tests needs to be done with respect to their possible dependence on the tested stations' local conditions. The observed profound differences between even close stations suggest that conclusions about the skill and suitability of time series analysis methods, obtained for a limited area or a single station, cannot be automatically generalized and applied to different locations. Noteworthy is the seasonal variance of the results, which implies the presence of a strong detectable nonlinear component in the relations between predictors and predictand in winter, and a rather linear character of these relations in summer.

It is also interesting that the effective nonlinearity is generally weaker for downscaling than for forecasting tasks (compare results in Figs. 5 and 8). This does not necessarily mean that the relations between predictors and predictand are intrinsically more linear in the case of downscaling. The reason for this difference may lie in the fact that while the forecasting tests were done solely on reanalysis data, which are relatively smooth by construction, downscaling predictands are local measurements. They reflect the situation in just a very small area, so it is possible for them to contain a stronger high-dimensional component, which may be nonlinear in its nature, but has a character of noise and is too complex to be described by any time series analysis method, even a nonlinear one.

\section{Conclusions}

Our primary intention was to make a comparison of three nonlinear methods and to identify the one most suitable for the analysis of climatic data at synoptic time scales. It turned out that the most commonly applied nonlinear method, multilayer perceptron neural network, may not necessarily be the best possible choice. The other two tested nonlinear techniques, method of local linear models and radial basis function neural network, performed equally well, even better in many cases, they do not suffer from MLPs' drawbacks, such as the problem of local minima of the error function or the danger of overtraining, and their implementation is relatively easy. We can therefore recommend them as a worthy alternative to MLPs.

The constructed maps of the geographical structure of errors and effective nonlinearity revealed profound spatial variations of the results. In case of prediction, a distinct contrast between the temperate zone (stronger nonlinearity) and the subtropical and tropical zones (weaker nonlinearity) was found, especially in summer. As for downscaling tests, we were not able to identify any apparent large-scale geographical distribution of nonlinearity, probably because the results for individual stations were influenced by the local conditions at least as much as by the large-scale climate dynamics. 
For both forecasting and downscaling, profound seasonal dependence of the results was observed, with errors being larger and nonlinearity being stronger in winter than in summer in most locations.

Acknowledgements. This work was supported by the Grant Agency of Charles University, grant 227/2002/B-GEO/MFF, Ministry of the Environment of the Czech Republic, grant VaV/740/2/03 and Research project 113200004 . We would also like to express our gratitude to B. Sivakumar for his valuable comments on the manuscript, and to thank the two anonymous reviewers of the previous version of the manuscript.

Edited by: A. Tsonis

Reviewed by: B. Sivakumar

\section{References}

Abarbanel, H. D. I.: Analysis of Observed Chaotic Data. Springer, New York, 272 pp., 1996.

Casaioli, M., Mantovani, R., Scorzoni, F. P., Puca, S., Speranza, A., and Tirozzi, B.: Linear and nonlinear post-processing of numerically forecasted surface temperature, Nonlin. Processes Geophys., 10, 373-383, 2003,

\section{SRef-ID: 1607-7946/npg/2003-10-373.}

Essenwanger, O. M.: General Climatology: Classification of Climates (World Survey of Climatology, Vol. 1 Part C), Elsevier, Amsterdam, 113 pp., 2001.

Farmer, J. D. and Sidorowich, J. J.: Predicting Chaotic Time Series, Phys. Rev. Lett., 59, 845-847, 1987, reprinted in Ott et al. (1994).

Fraedrich, K.: Estimating the Dimensions of Weather and Climate Attractors, J. Atmos. Sci., 43, 419-432, 1986.

Galka, A.: Topics in Nonlinear Time Series Analysis - With Implications for EEG Analysis, World Scientific Publishing, Singapore, 342 pp., 2000.

Hall, T., Brooks, H. E., and Doswell, C. A.: Precipitation Forecasting Using a Neural Network, Weather Forecast., 14, 338-345, 1999.

Haykin, S.: Neural networks: A comprehensive foundation (2nd edition), Prentice Hall, Upper Saddle River, New Jersey, 842 pp., 1999.

Hegger, R., Kantz, H., and Schreiber, T.: Practical implementation of nonlinear time series methods: The TISEAN package, CHAOS, 9, 413-435, 1999.

Huth, R.: Statistical Downscaling of Daily Temperature in Central Europe, J. Climate, 15, 1731-1742, 2002.

Huth, R.: Sensitivity of Local Daily Temperature Change Estimates to the Selection of Downscaling Models and Predictors, J. Climate, 17, 640-652, 2004.

Jayawardena, A. W. and Gurung, A. B.: Noise reduction and prediction of hydrometeorological time series: dynamical systems approach vs. stochastic approach, J. Hydrol., 228, 242-264, 2000.

Jayawardena, A. W., Li, W. K., and Xu, P.: Neighbourhood selection for local modelling and prediction of hydrological time series, J. Hydrol., 258, 40-57, 2002.

Kantz, H. and Schreiber, T.: Nonlinear time series analysis. Cambridge University Press, Cambridge, 304 pp., 1997.

Keppenne, C. L. and Nicolis, C.: Global Properties and Local Structure of the Weather Attractor over Western Europe, J. Atmos. Sci., 46, 2356-2370, 1989.
Kistler, R., Kalnay, E., Collins, W., Saha, S., White, G., Woollen, J., Chelliah, M., Ebisuzaki, W., Kanamitsu, M., Kousky, V., van den Dool, H., Jenne, R., and Fiorino, M.: The NCEP-NCAR 50Year Reanalysis: Monthly Means CD-ROM and Documentation, B. Am. Meteorol. Soc., 82, 247-268, 2001.

Klein Tank, A. M. G., Wijngaard, J. B., Können, G. P., et al.: Daily dataset of 20th-century surface air temperature and precipitation series for the European Climate Assessment, Int. J. Climatol., 22, 1441-1453, 2002.

MacQueen, J.: Some methods for classification and analysis of multivariate observations, Proceedings of the 5th Berkeley Symposium on Mathematical Statistics and Probability, 1, 281-297, 1967.

Marzban, C.: A Neural Network for Post-processing Model Output: ARPS, Mon. Weather Rev., 131, 1103-1111, 2003.

Miksovsky, J. and Raidl, A.: Testing for nonlinearity in European climatic time series by the method of surrogate data, Theor. Appl. Climatol., in press, 2005.

Ott, E., Sauer, T., and Yorke, J. A. (eds.): Coping with chaos: Analysis of chaotic data and the exploitation of chaotic systems, John Wiley \& Sons, New York, 418 pp., 1994.

Packard, H. N., Crutchfield, J. P., Farmer, J. D., and Shaw, R. S.: Geometry from a time series, Phys. Rev. Lett., 45, 712-716, 1980, reprinted in Ott et al. (1994).

Palus, M.: Detecting nonlinearity in multivariate time series, Phys. Lett. A, 213, 138-147, 1996.

Palus, M. and Novotna, D.: Testing for nonlinearity in weather records, Phys. Lett. A, 193, 67-74, 1994.

Pérez-Muñuzuri, V. and Gelpi, I. R.: Application of nonlinear forecasting techniques for meteorological modeling, Ann. Geophys., 18, 1349-1359, 2000,

SRef-ID: 1432-0576/ag/2000-18-1349.

Principe, J. C., Euliano, N. R., and Lefebvre, W. C.: Neural and Adaptive systems: Fundamentals through Simulations, John Wiley \& Sons, New York, 656 pp., 2000.

Sauer, T.: Time Series Prediction by Using Delay Coordinate Embedding., in: Time Series Prediction: Forecasting the Future and Understanding the Past, Santa Fe Institute Studies in the Science of Complexity XV, edited by: Weigend, A. S. and Gershenfeld, N. A., Addison-Wesley, Reading, 643 pp., 1993, reprinted in Ott et al. (1994)

Schoof, J. T. and Pryor, S. C.: Downscaling temperature and precipitation: A comparison of regression-based methods and artificial neural networks, Int. J. Climatol., 21, 773-790, 2001.

Schreiber, T. and Schmitz, A.: Surrogate time series, Physica D, 142, 346-382, 2000.

Shao, J.: Improving Nowcasts of Road Surface Temperature by a Backpropagation Neural Network, Weather Forecast., 13, 164171, 1998.

Sivakumar, B.: Chaos theory in geophysics: past, present and future, Chaos Soliton. Fract., 19, 441-462, 2004.

Sivakumar, B., Berndtsson, R., Olsson, J., Jinno, K., and Kawamura, A.: Dynamics of monthly rainfall-runoff process at the Göta basin: A search for chaos, Hydrol. Earth Syst. Sci., 4, $407-$ 417, 2000,

SRef-ID: 1607-7938/hess/2000-4-407.

Sugihara, G. and May, R. M.: Nonlinear forecasting as a way of distinguishing chaos from measurement error in time series, Nature, 344, 734-740, 1990, reprinted in Ott et al. (1994).

Takens, F.: Detecting Strange Attractors in Turbulence, in: Dynamical Systems and Turbulence, Lecture Notes in Mathematics, Vol. 898, edited by: Rand, D. A and Zouny, L. S., Springer, New 
York, 366-381, 1981.

Tang, B., Hsieh, W. W., Monahan, A. H., and Tangang, F. T.: Skill Comparisons between Neural Networks and Canonical Correlation Analysis in Predicting the Equatorial Pacific Sea Surface Temperatures, J. Climate, 13, 287-293, 2000.

Trigo, R. M. and Palutikof, J. P.: Simulation of daily temperatures for climate change scenarios over Portugal: a neural network model approach, Climate Res., 13, 45-59, 1999.

Tsonis, A. A.: Probing the linearity and nonlinearity in the transitions of the atmospheric circulation, Nonlin. Processes Geophys., 8, 341-345, 2001,

SRef-ID: 1607-7946/npg/2001-8-341 von Storch, H.: On the Use of "Inflation" in Statistical Downscaling, J. Climate, 12, 3505-3506, 1999.

Waelbroeck, H., López-Peña, R., Morales, T., and Zertuche, F.: Prediction of Tropical Rainfall by Local Phase Space Reconstruction, J. Atmos. Sci., 51, 3360-3364, 1994.

Weichert, A. and Bürger, G.: Linear versus nonlinear techniques in downscaling, Climate Res., 10, 83-93, 1998.

Wilks, D. S.: Statistical methods in the atmospheric sciences, Academic Press, San Diego, 467 pp., 1995. 